\title{
Improvement of the method for comparing subsidence of structures using the Fischer's F- test and the Foster-Stuart test
}

\author{
Abdusali Suyunov ${ }^{1, *}$, Shukhrat Suyunov ${ }^{1}$, Malika Aminjanova ${ }^{1}$ and Kamola \\ Rakhmatullaeva $^{1}$ \\ ${ }^{1}$ Department of Geodesy and Cartography, Samarkand State Architectural and Civil-Engineering \\ Institute, Lolazor 70, 140147, Samarkand city, Uzbekistan
}

\begin{abstract}
To improve the quality of construction and increase the durability of engineering structures under construction, complex geodetic works should be performed, including geodetic observations of deformations of structures. These observations are carried out during the construction of buildings and structures and their operation, mainly before the period of deformation stabilization. In this regard, a reliable statistical definition of deformations close to the limit is necessary, based on the data of geodetic observations. The research helps to improve the definition of deformations of structures using the Fischer's F-test and the Foster-Stuart test, based on analysis of the measurements of horizontal and vertical monitoring of industrial structures. According to the results, the magnitude of the subsidence plays a more significant role from than its absolute value, thus the value of the deformation intensity is of primary importance in justifying observation periodicity.
\end{abstract}

Keywords: deformation analysis, dispersion, Cochrane criterion, FosterStewart test, subsidence of structures, Fisher's F-test

\section{Introduction}

In General, the term "deformation" refers to a change in the shape of the object of observation. In geodesic practice, it is customary to consider deformation as a change in the position of an object relative to the original one.

Under constant pressure from the mass of the structure, the soils at the base of its foundation are gradually compacted (compressed) and a displacement occurs in the vertical plane, called the settlement of the structure. In addition to pressure from its own mass, subsidence (sediment) can be caused by other reasons: karst and landslide phenomena, changes in the level of groundwater, the work of heavy mechanisms, traffic, seismic phenomena, etc.

To improve the quality of construction and increase the durability of the engineering structures being erected, complex geodetic works should be carried out, including geodetic observations of the deformations of structures. These observations are carried out at the stage of construction of buildings and structures and at the stage of their operation, mainly

\footnotetext{
*Corresponding author: $\underline{\text { suyunov303@inbox.ru }}$
} 
before the period of deformation stabilization. In this regard, a reliable statistical determination of deformations is necessary, close to the limiting ones, according to the data of geodetic observations [5].

The method of performing geodetic works when observing subsidence and deformations of engineering structures is well studied and reflected in the works of Uzbek and foreign scientists geodesists $[1,2,4]$, as well as in the relevant regulatory documents $[7,8]$. According to most recent investigations, the precise observation of the subsidence (vertical monitoring) is very critical not only from the precision of the observation technology, but also from the variations of the environment, such as temporal variations of the reference horizontal level, or the geoid $[9,10]$.

In this article, vertical monitoring analysis, thus repeated measurements of subsidence of a construction are compared to each other is performed, using the Fisher's F and the Foster-Stewart tests, where the choice of time interval between the measurement campaigns depends on the type of structure, the period of its operation, the rate of deformation change among other factors.

On average, during the construction period, systematic observations are performed once or twice in a quarter, and during the operation period once or twice a year. In case of urgent observations, they are performed before and after the appearance of a factor that dramatically changes the usual course of deformation [1].

\section{Methodology}

The method considered in the article was used to analyse the results of geodetic observations of the subsidence of an industrial complex structure.

To determine a sufficient condition for the stability of a structure using Fisher's F-test, it is necessary to determine the standard deviation of the differences $\Delta(\mathrm{k})_{i}$ from the average unevenness

$$
m_{\Delta}^{2}=\sum_{i=1}^{\mathrm{n}-1}\left(\Delta(k)_{i}-\Delta(k)\right)^{2} /(n-2)
$$

and compare it with the general variance estimate

$$
\dot{m}^{2}=\sum_{i=1}^{n} m_{S i}^{2} / n
$$

According to Fisher's criterion [3]. The uneven settlement of the structure in this case is recognized as identified if at the level of significance $p$ the inequality is fulfilled [2]

$$
\left(m_{\Delta}^{2} / \dot{m}^{2}\right)>F_{1-p}
$$

where, $\mathrm{F}$ - distribution is considered with $\mathrm{f} 1=\mathrm{n}-1$ and $\mathrm{f} 2=\mathrm{n}$ degrees of freedom.

Expression (3) - one-way analysis of variance - is valid only when the general variance of reproducibility $\sigma 2$ is the same at all levels $n$. To test this assumption, it is necessary to consider the ratio of the maximum variance to the sum of all the others (Cochran's test) [4]

$$
g=m_{S i}^{2} / \sum_{k=1}^{n} m_{S \kappa}^{2}-m_{S i}^{2}
$$

The distribution of $g$ depends on $\mathrm{n}$ and $\mathrm{r}$ (the number of degrees of freedom - redundant measurements in the levelling network). In the case of a significant change in the estimates of the variances $\mathrm{m}^{2} \mathrm{~S}_{\mathrm{k}}(1 \leq k \leq p)$, e. $\mathrm{g}>\mathrm{g}_{\text {table }}$, it is necessary to select the appropriate transforming function [4]. 
Another useful criterion for identifying patterns in the differences in elevation or settlement of adjacent structural marks is the Foster-Stewart test [6]. A distinctive feature of this method is that it allows to simultaneously identify significant changes in variance estimates and determine possible trends on average. The test is based on two characteristics

$$
\begin{aligned}
& {[c]=\sum_{i=1}^{n} c_{i},} \\
& {[d]=\sum_{i=1}^{n} d_{i}}
\end{aligned}
$$

in which the values of $c_{i}$ and $d_{i}$ are calculated by the formulas

$$
\begin{aligned}
& c_{i}=u_{i}+l_{i}, \\
& d_{i}=u_{i}-l_{i}
\end{aligned}
$$

The $u_{i}$ and $l_{i}$ values are determined by sequentially comparing the benchmarks on structures, and

$$
u_{i}=\left\{\begin{array}{l}
1 \text { for } H_{i}>H_{i-1}, H_{i-2}, \ldots H_{l} \\
0-\text { in other cases }
\end{array}\right.
$$

$$
l_{i}=\left\{\begin{array}{l}
1 \text { for } H_{i}>H_{i-1,} H_{i-2}, \ldots H_{1}, \\
0 \text { - in other cases, }
\end{array}\right.
$$

When $c_{i} \in(0,1)$, and $c_{i}=0$ if $H_{i}$ is not an extreme among all previous values, in the opposite case $c_{i}=1$ [5], i.e.

$$
0 \leq[c] \leq n-1
$$

Comment 1. If the elevations (subsidence) of all points are equal, $\left(H_{1}=H_{2}=\ldots H_{n-1}=H_{n}\right.$ or $S_{1}=S_{2}=\ldots=S_{n-1}=S_{n}$ ), then $[c]=0$, if they change monotonically or their oscillations alternate, systematically increasing or decreasing, then

$$
[c]=n-1 .
$$

In turn, the quantity $d_{i} \in(0,1,-1)$, whence

$$
-(n-1) \leq[d] \leq n-1 .
$$

Comment 2. The lower limit corresponds to a monotonically decreasing, and the upper one - to a monotonically increasing series of elevation values (subsidence).

Cases are interesting for practice if the value $d=0$ : 
1) $H_{1}=H_{2}=\ldots=H_{n}\left(S_{1}=S_{2}=\ldots=S_{n-1}\right)$,

$$
\text { that } \sum_{i=1}^{n} u i=0, \quad \sum_{i=1}^{n} l i=0
$$

(which indicates the complete absence of uneven settlement of structures);

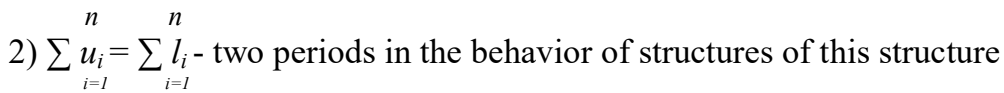

with opposite deformation tendencies;

3) $d=0$, when the motion (uplift or subsidence) of the $H_{i}\left(S_{i}\right)$ levels alternate [8]. It should be noted that the indices $[c]$ and $[d]$ are asymptotically normal and have distributions independent of each other (it is obvious that their distributions depend only on the order of arrangement of benchmarks on the structures) [7]. The indicator [c] is used in this case to statistically detect changes in variance, while [d] is used to determine possible trends in a trend. Testing the hypothesis about whether the differences $(d-0)$ and $(c-M)$ can be considered random ( $M$ is the expectation value of $c$ for a random distribution of benchmarks on a structure) is carried out using the Student's t-test

$$
t_{1-p}^{(2)}=\frac{[c]-M}{\sigma_{l}},
$$

where, the values $\sigma_{i}(i=1,2)$ are determined from the expressions

$$
\begin{gathered}
\sigma_{1} \approx \sqrt{2 \ln n-3.42532}, \\
\sigma_{2} \approx \sqrt{2 \ln n-0.8456}
\end{gathered}
$$

\section{Test and results}

The method introduced above was used to analyse the results of surveying/geodetic observations performed in order to moitor horizontal and/or vertical motions (deformation analysis) of industrial complex structures. In the frame of this study, subsidence of a pilefounded, reinforced concrete building has been performed.

Table 1. Initial values of research at this stage

\begin{tabular}{|c|c|c|c|c|c|c|c|c|}
\hline $\mathbf{H}$ & $\mathbf{M}$ & $\boldsymbol{\sigma}_{\mathbf{1}}$ & $\boldsymbol{\sigma}_{\mathbf{2}}$ & $\mathbf{H}$ & $\mathbf{M}$ & $\boldsymbol{\sigma}_{\mathbf{1}}$ & $\boldsymbol{\sigma}_{\mathbf{2}}$ \\
\hline 10 & 3,858 & 1,288 & 1,964 & & 26 & 5,672 & 1,800 & 2,379 \\
12 & 4,125 & 1,243 & 2,027 & 28 & 5,831 & 1,841 & 2,413 \\
14 & 4,392 & 1,361 & 2,105 & 30 & 5,990 & 1,882 & 2,447 \\
16 & 4,659 & 1,456 & 2,153 & 35 & 6,294 & 1,956 & 2,509 \\
18 & 4,927 & 1,535 & 2,216 & 40 & 6,557 & 2,019 & 2,561 \\
20 & 5,195 & 1,602 & 2,279 & 45 & 6,790 & 2,072 & 2,606 \\
22 & 5,354 & 1,660 & 2,313 & 50 & 6,998 & 2,121 & 2.645 \\
24 & 5,513 & 1,712 & 2,346 & 60 & 7,360 & 2,201 & 2,713 \\
\hline
\end{tabular}


The foundation inside the frame was built in the form of a monolithic reinforced concrete slab, lying on the soils of the poured base. The equipment installation technology has imposed strict requirements on the stability of the structure's base: the base roll tolerance was 0.001 .

As a preparatory analysis, the effect of the magnitude of the subsidence has been investigated by the Fischer's F-test. Assuming different amount of the subsidences, the values of $M, \sigma_{1}$ and $\sigma_{2}$ are estimated, and provided in Table 1 .

According to the results, the value of the subsidence intensity plays a more significant role from the point of view of judging the ongoing process than its absolute value, which leads to the conclusion that the value of the deformation intensity is of primary importance in justifying the accuracy of the observation periodicity.

Observations of deformations show that each individual tolerance value cannot serve as a consistent assessment of the stability of a structure. Therefore, for a complete judgment about the stability of the structure, the observation technique must be developed based on the complex effect of all characteristics that have limiting values.

\section{References}

1. V. I. Bandurka, N. A. Loshkarev, J.Geodesy and aerial photography, 4 (1972)

2. P. I. .Bright, E. N. Medvetsky, Measurements of sediments and deformations by geodesic methods. (1959)

3. T. A. Gura, A. O. Biryukova, J. Young scientist. 30 (2016)

4. E. I. Pustylnik, Statistical methods of analysis and processing of observations, (1966)

5. A. S. Suyunov, M.B. Aminjanova et al., ICT youth and their social well-being (2013)

6. E. M. Chetyrkin, Statistical methods of forecasting (1975)

7. GOST 8.207-76. GSI. Direct measurements with multiple observations. Methods of processing the results of observations. Fundamentals.

8. Guide to observations of deformations of the foundations of buildings and structures. (Moscow, 1975)

9. Cs. Égetö, L. Földváry, T. Huszák, The effect of tunnelling on repeated precise levelling measurements for vertical deformation control of the Metro4 project, Journal of Geodetic Science, 3(2): 95-102 (2013)

10. Cs. Égető, N. Rehány, L. Földváry, Variations of the gravity field due to excavations of the Budapest Metro4 subway line, Periodica. Polytechnica Civil Engineering, 58(2): $131-136$ (2014) 